\title{
On a Set of the First Category
}

\author{
Hein Putter ${ }^{1}$ \\ Willem R. van Zwet $^{2}$
}

\begin{abstract}
In an analysis of the bootstrap Putter \& van Zwet (1993) showed that under quite general circumstances, the bootstrap will work for "most" underlying distributions. In fact, the set of exceptional distributions for which the bootstrap does not work was shown to be a set $D$ of the first category in the space $\mathcal{P}$ of all possible underlying distributions, equipped with a topology $\Pi$. Such a set of the first category is usually "small" in a topological sense. However, it is known that this concept of smallness may sometimes be deceptive and in unpleasant cases such "small" sets may in fact be quite large.

Here we present a striking and hopefully amusing example of this phenomenon, where the "small" subset $D$ equals all of $\mathcal{P}$. We show that as a result, a particular version of the bootstrap for the sample minimum will never work, even though our earlier results tell us that it can only fail for a "small" subset of underlying distributions. We also show that when we change the topology on $\mathcal{P}$-and as a consequence employ a different resampling distribution - this paradox vanishes and a satisfactory version of the bootstrap is obtained. This demonstrates the importance of a proper choice of the resampling distribution when using the bootstrap.
\end{abstract}

\subsection{Introduction}

Many of the results of asymptotic statistics cannot be established in complete generality. One often has to allow the possibility that the result will not hold if the underlying probability distribution belongs to a small subset $D$ of the collection of all possible underlying probability distributions $\mathcal{P}$. In many concrete examples, $D$ will turn out to be empty, but in general one has to take the existence of such an exceptional set into account.

If $\mathcal{P}$ is a parametric model, the exceptional set $D$ will typically be small in the sense that it is indexed by a set of Lebesgue measure zero in the Euclidean parameter space. From a technical point of view, its occurrence is caused by an application of a result like Egorov's or Lusin's theorem where exceptional sets of arbitrarily small Lebesgue measure occur. In more general models one could conceivably use similar tools for more general

\footnotetext{
${ }^{1}$ University of Leiden

${ }^{2}$ University of Leiden and University of North Carolina.
} 
measures, but it is difficult to think of a measure on $\mathcal{P}$ which is such that we can agree that a set of measure zero is indeed small in a relevant sense.

In a recent study of resampling, we have followed a different path and established asymptotic results where the exceptional set is small in a topological rather than a measure-theoretic sense (Putter \& van Zwet 1993). If we equip the set $\mathcal{P}$ with a metric $p$, the exceptional set $D$ in these results is a set of the first category in the metric space $(\mathcal{P}, p)$. We recall that a set of the first category is a countable union of nowhere dense sets, and that a set is nowhere dense if its closure has empty interior. Equivalently, a set is of the first category if it can be covered by a countable union of closed sets, each of which has empty interior.

This concept of a small set was used by Le Cam as early as Le Cam (1953), where it is shown that superefficiency can only occur on a set of the first category. In a parametric setting, Le Cam was careful to point out that under the right conditions the exceptional set also corresponds to a set of Lebesgue measure zero in the parameter space. The same is true for the results in Putter \& van Zwet (1993), as shown by Putter (1994).

Of course the question remains whether a set of the first category is indeed small in any accepted sense. If $(\mathcal{P}, p)$ is complete, we know that a set of the first category is small, for example in the sense that it has a dense complement (cf. Dudley 1989, pp. 43-44). If ( $\mathcal{P}, p)$ is not complete, then a set of the first category can be uncomfortably large: in fact we shall see that the entire space $\mathcal{P}$ may be of the first category itself.

In this note we discuss a particular statistical model $\mathcal{P}_{0}$ equipped with Hellinger metric $H$, such that $\left(\mathcal{P}_{0}, H\right)$ is not complete and $\mathcal{P}_{0}$ is of the first category in $\left(\mathcal{P}_{0}, H\right)$. An application of our results on resampling shows that a particular version of the bootstrap will work except if the underlying distribution belongs to a set $D$ of the first category. Unfortunately, it turns out that $D=\mathcal{P}_{0}$ so that we have no guarantee that this version of the bootstrap will ever work, and indeed it may never do. Luckily, our analysis also shows that we need not despair. It turns out that our problems are not caused by any inherent pathology of the model $\mathcal{P}_{0}$, but by a wrong choice of metric on $\mathcal{P}_{0}$. If we replace $H$ by a different, complete, metric and modify the construction of the bootstrap accordingly, the pathology disappears and we obtain a version of the bootstrap that will work for any $P \in \mathcal{P}_{0}$. In fact the example may serve to clarify the importance of a correct choice of the resampling distribution when using the bootstrap.

In Section 2 we exhibit the particular class of distributions $\mathcal{P}_{0}$ which is of the first category in $\left(\mathcal{P}_{0}, H\right)$. In section 3 we show that this class is not an artificial construct, but that it is the natural model for a statistical situation of interest. We then proceed to make the connection with a result on the bootstrap in Putter \& van Zwet (1993) and show that this result doesn't produce a satisfactory version of the bootstrap for this model. Finally we show that a different choice of metric on $\mathcal{P}_{0}$ will resolve our problems. 


\subsection{A set of the first category}

Let us consider the class $\mathcal{P}_{0}$ of probability distributions $P$ on $(0, \infty)$ which have distribution functions $F$ satisfying

$$
\lim _{x \downarrow 0} \frac{F(x)}{x}=a(P) \in(0, \infty) .
$$

We equip $\mathcal{P}_{0}$ with Hellinger metric $H$. For distributions $P, Q \in \mathcal{P}_{0}$, with densities $f$ and $g$ with respect to a common $\sigma$-finite measure $\nu$, this is defined by

$$
H(P, Q)^{2}=\int(\sqrt{f}-\sqrt{g})^{2} d \nu
$$

(2) Proposition The set $\mathcal{P}_{0}$ is of the first category in $\left(\mathcal{P}_{0}, H\right)$.

Proof. For $k=1,2, \ldots$, let $\delta_{k}=1 / k$ and

$$
B_{k}=\left\{P \in \mathcal{P}_{0}:\left|\frac{F(x)}{x}-\frac{F\left(\delta_{k}\right)}{\delta_{k}}\right| \leq 1 \text { for } 0<x \leq \delta_{k}\right\} .
$$

Clearly, $\mathcal{P}_{0} \subset \bigcup_{k=1}^{\infty} B_{k}$, and since convergence in Hellinger metric implies pointwise convergence of distribution functions, we see that each $B_{k}$ is closed in $\left(\mathcal{P}_{0}, H\right)$. It remains to be shown that no $B_{k}$ contains an open set.

Fix $k$ and choose a distribution $P \in B_{k}$ with distribution function $F$ and with $a(P)=\alpha$. Define $G_{n}(x)=\min \left(n^{-1},(3+\alpha) x\right), F_{n}=\max \left(G_{n}, F\right)$, and let $P_{n}$ be the distribution with distribution function $F_{n}$. Then $a\left(P_{n}\right)=$ $3+\alpha$ but, for $n$ large enough, $F_{n}\left(\delta_{k}\right) / \delta_{k}=F\left(\delta_{k}\right) / \delta_{k} \leq 1+\alpha$ because $P \in B_{k}$. It follows that $P_{n} \notin B_{k}$ for large $n$, even though $P_{n}$ converges to $P$ in Hellinger metric.

\subsection{A bootstrap fiasco}

Let $\mathcal{P}$ be a class of probability distributions on $\mathbb{R}$. We equip $\mathcal{P}$ with a metric $p$. Let $X_{1}, X_{2}, \ldots$ be independent and identically distributed (i.i.d.) random variables with (unknown) common distribution $P \in \mathcal{P}$. We are interested in the large sample behavior of a random variable

$$
Y_{N}=y_{N}\left(X_{1}, \ldots, X_{N} ; P\right) \text {. }
$$

Let $\tau_{N}(P)$ denote the distribution of $Y_{N}$ under $P \in \mathcal{P}$, and suppose that, for every $P \in \mathcal{P}, \tau_{N}(P)$ converges weakly to a limit distribution $\tau(P)$. If $\widehat{P}_{N}=P_{N}\left(X_{1}, \ldots, X_{N}\right)$ is an estimator of $P$ taking values in $\mathcal{P}$, then $\tau_{N}\left(\widehat{P}_{N}\right)$ is called a bootstrap estimator of $\tau_{N}(P)$, or of $\tau(P)$, with resampling distribution $\widehat{P}_{N}$. For all $P$ and $\widehat{P}_{N}$, the distributions $\tau_{N}(P)$, $\tau(P)$, and $\tau_{N}\left(\widehat{P}_{N}\right)$ are elements of the class $\mathcal{R}$ of all probability measures on $\mathbb{R}$. We equip this class with Lévy distance $\ell$, or any other metric which 
metrizes weak convergence. The bootstrap is said to work for a particular $P \in \mathcal{P}$ if it is an $\ell$-consistent estimator of $\tau_{N}(P)$, i.e. if $\ell\left(\tau_{N}\left(\widehat{P}_{N}\right), \tau_{N}(P)\right)$ converges to zero in probability under $P$. As $\ell\left(\tau_{N}(P), \tau(P)\right) \rightarrow 0$, this is the same as $\ell$-consistency for estimating the limit distribution $\tau(P)$.

The following two propositions are taken from Putter \& van Zwet (1993).

(4) Proposition Suppose that

(i) The sequence of maps $\tau_{N}:(\mathcal{P}, p) \rightarrow(\mathcal{R}, \ell)$ is equicontinuous on $\mathcal{P}$;

(ii) $\widehat{P}_{N}$ takes values in $\mathcal{P}$ and is a p-consistent estimator of $P$, i.e. $p\left(\widehat{P}_{N}, P\right) \stackrel{P}{\rightarrow} 0$ for every $P \in \mathcal{P}$.

Then the bootstrap $\tau_{N}\left(\widehat{P}_{N}\right)$ works for every $P \in \mathcal{P}$.

(5) Proposition Suppose that

(i) $\tau_{N}:(\mathcal{P}, p) \rightarrow(\mathcal{R}, \ell)$ is continuous for every $N$;

(ii) For every $P \in \mathcal{P}, \tau_{N}(P)$ converges weakly to a limit $\tau(P)$;

(iii) $\widehat{P}_{N}$ takes values in $\mathcal{P}$ and is a p-consistent estimator of $P$, i.e. $p\left(\widehat{P}_{N}, P\right) \stackrel{P}{\rightarrow} 0$ for every $P \in \mathcal{P}$.

Then there exists a set $D$ of the first category in $(\mathcal{P}, p)$ such that the sequence $\tau_{N}$ is equicontinuous at every $P \in \mathcal{P} \backslash D$ and hence the bootstrap $\tau_{N}\left(\widehat{P}_{N}\right)$ works for every $P \in \mathcal{P} \backslash D$.

Usually these results are used with Hellinger distance $H$ for $p$, and on closer inspection it often turns out that the exceptional set $D$ may be taken to be empty.

In the remainder of this paper we shall consider a specific example of this situation. We choose $\mathcal{P}=\mathcal{P}_{0}$, the class of distributions defined in (1). For i.i.d. random variables $X_{1}, \ldots, X_{N}$ taking values in $(0, \infty)$ with common distribution $P \in \mathcal{P}_{0}$, we define

$$
Y_{N}^{0}=N \min \left\{X_{1}, \ldots, X_{N}\right\} .
$$

Note that $\mathcal{P}_{0}$ is a natural model for studying the large sample behavior of $Y_{N}^{0}$, since it is precisely the class of underlying distributions for which the distributions $\tau_{N}(P)$ of $Y_{N}^{0}$ under $P$ converge weakly to a non-degenerate limit, which is an exponential distribution with parameter $a(P)$.

Bootstrapping the sample minimum is a problem of some notoriety as it is an early example where the usual choice of the empirical distribution $P_{N}$ for the resampling distribution $\widehat{P}_{N}$ does not work. To check whether the bootstrap with a different choice of $\widehat{P}_{N}$ will work for "most" $P \in \mathcal{P}_{0}$, we may appeal to Proposition 5. In doing so, we are still free to choose a metric $p$ on $\mathcal{P}_{0}$ and we shall make the usual choice by taking $p$ to be Hellinger distance $H$. Since $Y_{N}^{0}$ is a function of $X_{1}, \ldots, X_{N}$ only, and not of $P$, it is easy to see that $\tau_{N}:\left(\mathcal{P}_{0}, H\right) \rightarrow(\mathcal{R}, \ell)$ is continuous for each $N$. As $\tau_{N}(P)$ converges weakly to a limit $\tau(P)$ for every $P \in \mathcal{P}_{0}$, Proposition 5 asserts that if $\widehat{P}_{N}$ is a Hellinger consistent estimator with values in $\mathcal{P}_{0}$, then the bootstrap $\tau_{N}\left(\widehat{P}_{N}\right)$ will work except for $P$ in a set 
$D$ of the first category in $\left(\mathcal{P}_{0}, H\right)$. The content of Proposition 2 having made us somewhat suspicious, we may want to investigate the nature of the exceptional set $D$ where the functions $\tau_{N}:\left(\mathcal{P}_{0}, H\right) \rightarrow(\mathcal{R}, \ell)$ are not equicontinuous. Since $\tau(P)$ depends on $P$ only through $a(P)$, and any $P \in \mathcal{P}_{0}$ may be approximated arbitrarily well in Hellinger distance by a sequence $P_{r} \in \mathcal{P}_{0}$ with a constant value of $a\left(P_{r}\right)$ different from $a(P)$, we know that the limit distribution $\tau$ is nowhere continuous in $P$. This implies that the functions $\tau_{N}$ are not equicontinuous at any $P \in \mathcal{P}_{0}$, so that our worst suspicions are confirmed: the exceptional set $D$ equals the entire set of possible distributions in this case. Our application of Proposition 5 with $p=H$ has therefore produced no positive information concerning this example at all.

Even though Proposition 5 is vacuous in this case, it might still by a stroke of luck be true that the bootstrap estimate $\tau_{N}\left(\widehat{P}_{N}\right)$ would work for most reasonable Hellinger-consistent estimators $\widehat{P}_{N}$ of $P$. First of all we note that it is indeed possible to construct an estimator of $P$ which is Hellinger-consistent for every distribution $P$ on $\mathbb{R}$ which has no singular part (cf. Devroye \& Györfi 1990, p. 1497). All we have to do is to assign probability $k / N$ to all values which were observed $k>1$ times, and add a kernel density estimator based on the remaining values which have only been observed once. Using the normal kernel we arrive at an estimator $F_{N}^{*}$ for the distribution function $F$ of $P$ which is given by

$$
F_{N}^{*}(x)=\frac{1}{N} \sum_{i=1}^{N}\left(\delta_{i} 1_{(0, x]}\left(X_{i}\right)+\left(1-\delta_{i}\right) \Phi\left(\frac{x-X_{i}}{h_{N}}\right)\right)
$$

where $\Phi$ is the standard normal distribution function,

$$
\delta_{i}= \begin{cases}0 & \text { if } X_{j} \neq X_{i} \text { for } j \neq i, \\ 1 & \text { otherwise }\end{cases}
$$

and $h_{N} \rightarrow 0$ but $N h_{N} \rightarrow \infty$. Admittedly, $F_{N}^{*}$ does not satisfy (1) and hence the corresponding estimator $P_{N}^{*}$ of $P$ does not take its values in $\mathcal{P}_{0}$ as is required in Proposition 5. However this defect is easily cured by considering the following slight modification of $F_{N}^{*}$,

$$
\widehat{F}_{N}(x)= \begin{cases}x F_{N}^{*}\left(M_{N}\right) / M_{N} & \text { for } 0 \leq x<M_{N}, \\ F_{N}^{*}(x) & \text { for } x \geq M_{N},\end{cases}
$$

where $M_{N}=\min \left(X_{1}, \ldots, X_{N}\right)$. Clearly $\widehat{F}_{N}$ satisfies (1), and hence the corresponding estimator $\widehat{P}_{N}$ of $P$ takes its values in $\mathcal{P}_{0}$ and is Hellinger consistent for every $P \in \mathcal{P}_{0}$ which has no singular part. Nevertheless we shall see that the bootstrap $\tau_{N}\left(\widehat{P}_{N}\right)$ based on this estimator does not work for any $P \in \mathcal{P}_{0}$.

The bootstrap $\tau_{N}\left(\widehat{P}_{N}\right)$ has distribution function

$$
\widehat{H}_{N}(y)=1-\left(1-\widehat{F}_{N}(y / N)\right)^{N} \text {. }
$$


For $P \in \mathcal{P}_{0}$, the limit distribution $\tau(P)$ is exponential with parameter $a(P) \in(0, \infty)$, and hence the bootstrap $\tau_{N}\left(\widehat{P}_{N}\right)$ will work for a particular $P \in \mathcal{P}_{0}$ if and only if

$$
\sup _{y>0}\left|\widehat{H}_{N}(y)-[1-\exp \{-a(P) y\}]\right| \stackrel{P}{\longrightarrow} 0 .
$$

This is easily seen to be equivalent to

$$
N \widehat{F}_{N}\left(\frac{y}{N}\right)-a(P) y \stackrel{P}{\rightarrow} 0
$$

for every $y>0$.

However, (10) cannot hold. If $F_{N}$ denotes the empirical distribution function, (7) implies that for all $x$,

$$
F_{N}^{*}(x) \geq \frac{1}{N} \sum_{i=1}^{N} 1_{(0, x]}\left(X_{i}\right)\left[\delta_{i}+\frac{1-\delta_{i}}{2}\right] \geq 1 / 2 F_{N}(x)
$$

As $F_{N}(x)=0$ for $x<M_{N}$, we also find that for all $x$,

$$
\widehat{F}_{N}(x) \geq 1 / 2 F_{N}(x) \text {. }
$$

Hence, for every $y>0$, the definition (1) ensures that as $N \rightarrow \infty$,

$$
\begin{gathered}
P\left(\left|N \widehat{F}_{N}\left(\frac{y}{N}\right)-a(P) y\right| \geq a(P) y\right) \geq P\left(N \widehat{F}_{N}\left(\frac{y}{N}\right) \geq 2 a(P) y\right) \\
\quad \geq P\left(N F_{N}\left(\frac{y}{N}\right) \geq 4 a(P) y\right)=P(Z \geq 4 a(P) y)+o(1) \not 0,
\end{gathered}
$$

where $Z$ has a Poisson distribution with expectation $a(P) y>0$. This shows that (10) is false, and as a consequence, the bootstrap based on $\widehat{P}_{N}$ does not work for any $P \in \mathcal{P}_{0}$, and the fiasco is indeed complete.

\subsection{A bootstrap success}

Luckily, the disastrous results of the previous section also indicate quite clearly how the damage may be repaired. Our problems in the previous section originate from the fact that the parameter of the exponential limit distribution $a(P)$ is not a continuous function of the underlying distribution $P \in \mathcal{P}_{0}$ with respect to Hellinger distance on $\mathcal{P}_{0}$. Hence we should look for a different metric on $\mathcal{P}_{0}$, and in view of the definition of $a(P)$ in (1), one obvious candidate is a metric $\pi$ defined by

$$
\pi(P, Q)=\sup _{x>0} \frac{|F(x)-G(x)|}{x}
$$

where $F$ and $G$ denote the distribution functions corresponding to $P$ and $Q$.

With this new metric $\pi$, things immediately fall into place. The metric space $\left(\mathcal{P}_{0}, \pi\right)$ is easily seen to be complete and hence sets of the first category have dense complements. Clearly the exponential limit distribution 
$\tau(P)$ is continuous when viewed as a map $\tau:\left(\mathcal{P}_{0}, \pi\right) \rightarrow(\mathcal{R}, \ell)$. Also, the sequence of distributions $\tau_{N}(P)$ of $Y_{N}$ is equicontinuous on $\mathcal{P}_{0}$. To see this, note that for underlying distributions $P$ and $Q$ with distribution functions $F$ and $G, Y_{N}$ has distribution functions

$$
H_{N, P}(y)=1-(1-F(y / N))^{N}
$$

and

$$
H_{N, Q}(y)=1-(1-G(y / N))^{N} .
$$

Fix $P \in \mathcal{P}_{0}$ and $0<\epsilon<1$. Choose positive numbers $y_{0}$ and $z_{0}$ such that

$$
y_{0}=\frac{4 \log (4 / \epsilon)}{a(P)} \text { and } F(z) \geq 1 / 2 a(P) z \quad \text { for } 0 \leq z \leq z_{0}
$$

and note that $\left|a^{N}-b^{N}\right| \leq N|a-b|$ if $0 \leq a, b \leq 1$. If $N \geq y_{0} / z_{0}$ we choose $\pi(P, Q) \leq \frac{\epsilon}{2 y_{0}} \leq \frac{a(P)}{4}$ and find

$$
\begin{aligned}
\sup _{y} \mid & H_{N, P}(y)-H_{N, Q}(y)\left|=\sup _{y}\right|(1-F(y / N))^{N}-(1-G(y / N))^{N} \mid \\
& \leq N \sup _{y \leq y_{0}}|F(y / N)-G(y / N)|+\left(1-F\left(y_{0} / N\right)\right)^{N}+\left(1-G\left(y_{0} / N\right)\right)^{N} \\
& \leq y_{0} \pi(P, Q)+\exp \left\{-N F\left(y_{0} / N\right)\right\}+\exp \left\{-N G\left(y_{0} / N\right)\right\} \\
& \leq \frac{\epsilon}{2}+\exp \left\{-1 / 2 a(P) y_{0}\right\}+\exp \left\{-1 / 2 a(P) y_{0}+y_{0} \pi(P, Q)\right\} \\
& \leq \frac{\epsilon}{2}+\left(\frac{\epsilon}{4}\right)^{2}+\exp \left\{-1 / 4 a(P) y_{0}\right\}=\frac{\epsilon}{2}+\frac{\epsilon^{2}}{16}+\frac{\epsilon}{4}<\epsilon .
\end{aligned}
$$

On the other hand, if $1 \leq N<y_{0} / z_{0}$, we choose $y_{1}$ such that

$$
1-F\left(\frac{z_{0} y_{1}}{y_{0}}\right) \leq \frac{\epsilon}{4} \text {. }
$$

For $\pi(P, Q) \leq \frac{\epsilon}{4 y_{1}}$ we find

$$
\begin{aligned}
\sup _{y} \mid H_{N, P} & (y)-H_{N, Q}(y) \mid \\
& \leq y_{1} \pi(P, Q)+\left(1-F\left(y_{1} / N\right)\right)^{N}+\left(1-G\left(y_{1} / N\right)\right)^{N} \\
& \leq \frac{\epsilon}{4}+\left(1-F\left(y_{1} / N\right)\right)+\left(1-F\left(y_{1} / N\right)+\frac{y_{1}}{N} \pi(P, Q)\right) \\
& \leq \frac{\epsilon}{4}+\left(1-F\left(\frac{z_{0} y_{1}}{y_{0}}\right)\right)+\left(1-F\left(\frac{z_{0} y_{1}}{y_{0}}\right)+\frac{\epsilon}{4}\right) \leq \epsilon .
\end{aligned}
$$

Hence for every $0<\epsilon<1$ there exists $\delta>0$ depending on $P$ but not on $Q$, such that $\pi(P, Q) \leq \delta$ implies $\sup _{y}\left|H_{N, P}(y)-H_{N, Q}(y)\right| \leq \epsilon$ for all $N$, which establishes the equicontinuity of $\left\{\tau_{N}\right\}$ on $\mathcal{P}_{0}$.

By Proposition 4 the equicontinuity of $\tau_{N}:\left(\mathcal{P}_{0}, \pi\right) \rightarrow(\mathcal{R}, \ell)$ implies that the bootstrap $\tau_{N}\left(\widetilde{P}_{N}\right)$ will work for all $P \in \mathcal{P}_{0}$ if $\widetilde{P}_{N}$ is a $\pi$-consistent estimator of $P$. An example of such an estimator is the random distribution 
$\widetilde{P}_{N}$ with distribution function

$$
\widetilde{F}_{N}(x)= \begin{cases}x F_{N}\left(\xi_{N}\right) / \xi_{N} & \text { for } 0 \leq x<\xi_{N} \\ F_{N}(x) & \text { for } x \geq \xi_{N}\end{cases}
$$

where $F_{N}$ denotes the empirical distribution function, $\xi_{N} \rightarrow 0$ but $N \xi_{N} \rightarrow$ $\infty$. To see this, let $F$ denote the distribution function corresponding to the underlying distribution $P \in \mathcal{P}_{0}$. Then $F_{N}\left(\xi_{N}\right) / \xi_{N} \stackrel{P}{\rightarrow} a(P)$ if $\xi_{N} \rightarrow 0$ and $N \xi_{N} \rightarrow \infty$, since

$$
E \frac{F_{N}\left(\xi_{N}\right)}{\xi_{N}}=\frac{F\left(\xi_{N}\right)}{\xi_{N}} \rightarrow a(P)
$$

and

$$
\sigma^{2}\left(\frac{F_{N}\left(\xi_{N}\right)}{\xi_{N}}\right)=\frac{F\left(\xi_{N}\right)\left(1-F\left(\xi_{N}\right)\right)}{N \xi_{N}^{2}} \rightarrow 0
$$

It follows that, if $\xi_{N} \rightarrow 0$ and $N \xi_{N} \rightarrow \infty$ as $N \rightarrow \infty$, then

$$
\sup _{x \leq \xi_{N}} \frac{\left|\widetilde{F}_{N}(x)-F(x)\right|}{x} \leq\left|\frac{F_{N}\left(\xi_{N}\right)}{\xi_{N}}-a(P)\right|+\sup _{x \leq \xi_{N}}\left|\frac{F(x)}{x}-a(P)\right| \stackrel{P}{\rightarrow} 0 .
$$

Also, for every sequence $\eta_{N} \rightarrow 0$ with $\eta_{N}>\xi_{N}$,

$$
\sup _{\xi_{N} \leq x \leq \eta_{N}} \frac{\left|\widetilde{F}_{N}(x)-F(x)\right|}{x}=\sup _{\xi_{N} \leq x \leq \eta_{N}} \frac{\left|F_{N}(x)-F(x)\right|}{F(x) / a(P)}+o(1)
$$

and this tends to zero in probability if $N \xi_{N} \rightarrow \infty$ (cf. Chang 1955, Theorem 1; see also Shorack \& Wellner 1986, p. 424). Finally, taking $\eta_{N}$ such that $N^{1 / 2} \eta_{N} \rightarrow \infty$, we have

$$
\sup _{x>\eta_{N}} \frac{\left|F_{N}(x)-F(x)\right|}{x} \leq \sup _{x} \frac{\left|F_{N}(x)-F(x)\right|}{\eta_{N}}=O_{P}\left(N^{-1 / 2} \eta_{N}^{-1}\right) .
$$

Thus the $\pi$-consistency of $\widetilde{F}_{N}$ follows and we have shown

(13) Proposition If $\widetilde{P}_{N}$ is an estimator of $P$ with distribution function $\widetilde{F}_{N}$ given by (12), then the bootstrap estimator $\tau_{N}\left(\widetilde{P}_{N}\right)$ of the distribution $\tau_{N}(P)$ of $Y_{N}^{0}$ is consistent for all $P \in \mathcal{P}_{0}$.

Acknowledgments: This research was supported by the Netherlands' Organization for Scientific Research (NWO) and by the Sonderforschungsbereich 343 "Diskrete Strukturen in der Mathematik" at the University of Bielefeld, German Federal Republic.

We are grateful to David Pollard for his careful reading of this paper which led to many improvements. 


\subsection{REFERENCES}

Chang, L.-C. (1955), 'On the ratio of the empirical distribution to the theoretical distribution function', Acta Math. Sinica 5, 347368. (English Translation in: Selected Translations in Mathematical Statististics and Probability, 4, 17-38 (1964).).

Devroye, L. \& Győrfi, L. (1990), 'No empirical probability measure can converge in the total variation sense for all distributions', Annals of Statistics 18, 1496-1499.

Dudley, R. M. (1989), Real Analysis and Probability, Wadsworth, Belmont, California.

Le Cam, L. (1953), 'On some asymptotic properties of maximum likelihood estimates and related Bayes estimates', University of California Publications in Statistics 1, 277-330.

Putter, H. (1994), Consistency of Resampling Methods, PhD thesis, University of Leiden.

Putter, H. \& van Zwet, W. R. (1993), Consistency of plug-in estimators with application to the bootstrap, Technical report, University of Leiden.

Shorack, G. R. \& Wellner, J. A. (1986), Empirical Processes with Applications to Statistics, Wiley, New York. 